\title{
Meet and Join within the Lattice of Set Partitions
}

\author{
E. Rodney Canfield \\ Department of Computer Science \\ The University of Georgia \\ Athens, GA 30602 USA \\ erca@cs.uga.edu
}

Submitted: January 21, 2001; Accepted: March 1, 2001.

MR Subject Classifications: 05A18, 05A15, 05A16, 06C10

\begin{abstract}
We build on work of Boris Pittel [5] concerning the number of $t$-tuples of partitions whose meet (join) is the minimal (maximal) element in the lattice of set partitions.
\end{abstract}

\section{Introduction}

Recall that a partition of the set $[n]=\{1,2, \ldots n\}$ is a collection of nonempty, pairwise disjoint subsets of $[n]$ whose union is $[n]$. The subsets are called blocks. One partition $\pi_{1}$ is said to refine another $\pi_{2}$, denoted $\pi_{1} \leq \pi_{2}$, provided every block of $\pi_{1}$ is contained in some block of $\pi_{2}$. The refinement relation is a partial ordering of the set $\Pi_{n}$ of all partitions of $[n]$. Given two partitions $\pi_{1}$ and $\pi_{2}$, their meet, $\pi_{1} \wedge \pi_{2}$, (respectively join, $\pi_{1} \vee \pi_{2}$ ) is the largest (respectively smallest) partition which refines (respectively is refined by) both $\pi_{1}$ and $\pi_{2}$. The meet has as blocks all nonempty intersections of a block from $\pi_{1}$ with a block from $\pi_{2}$. The blocks of the join are the smallest subsets which are exactly a union of blocks from both $\pi_{1}$ and $\pi_{2}$. Under these operations, the poset $\Pi_{n}$ is a lattice.

Recently Pittel has considered the number $M_{n}^{(t)}$ of $t$-tuples of partitions whose meet is the minimal partition $\{\{1\},\{2\}, \ldots\{n\}\}$, and $J_{n}^{(t)}$ the number of $t$-tuples whose join is the maximal partition $\{\{1,2, \ldots, n\}\}$. We shall prove

Theorem 1 Let $M_{t}(x)$ and $J_{t}(x)$ be the exponential generating functions for the sequences $M_{n}^{(t)}$ and $J_{n}^{(t)}$. Then

$$
M_{t}\left(e^{x}-1\right)=\sum_{n=0}^{\infty}\left(B_{n}\right) \frac{x^{n}}{n !}=\exp \left\{J_{t}(x)-1\right\} .
$$

where $B_{n}$ is the $n$-th Bell number, the total number of partitions of the set $[n]$. 
Remark. What about $n=0$ and/or $t=0$ ? The lattice $\Pi_{0}$ has exactly one element, and thus is isomorphic to $\Pi_{1}$. Generally, one takes the empty meet to be the maximal element and the empty join to be the minimal element. Thus, there is some logical justification to define

$$
\begin{aligned}
& M_{0}^{(t)}=J_{0}^{(t)}=1 \text { for all } t \\
& M_{n}^{(0)}=J_{n}^{(0)}=1 \text { for } n=0,1 \\
& M_{n}^{(0)}=J_{n}^{(0)}=0 \text { for all } n \geq 2
\end{aligned}
$$

In particular, $M_{0}(x)=J_{0}(x)=1+x$, and $M_{1}(x)=J_{1}(x)=e^{x}$. These latter two when inserted in the theorem yield immediately recognized identities.

To prove the first equality of Theorem 1 we shall use the following known result:

Theorem 2 Let $E_{n}$ be the edge set of the complete graph $K_{n}, G_{S}$ the graph with vertex set $[n]$ and edge set $S \subseteq E_{n}$, and $\mathrm{c}(G)$ the number of connected components in the graph G. Then,

$$
\sum_{S \subseteq E_{n}}(-1)^{|S|} X^{\mathrm{c}\left(G_{S}\right)}=X(X-1) \cdots(X-n+1) .
$$

A consequence of Theorem 2 is our later formula (2) which gives $M_{n}^{(t)}$ as a sum of products of Bell number powers with Stirling numbers of the first kind. With the second equality of Theorem 1 we can prove

\section{Theorem 3}

$$
J_{n}^{(2)}=\left(B_{n}\right)^{2} \times\left(1-\frac{r^{2}}{n}-\frac{2 r^{3}+2 r^{4}+2 r^{5}+r^{6}}{(r+1)^{2} n^{2}}+\mathrm{O}\left(r^{7} / n^{3}\right)\right)
$$

where $r$ is the positive real solution of the equation $r e^{r}=n$.

This improves on Pittel's estimate that $J_{n}^{(t)}$ is $\left(B_{n}\right)^{t}\left(1+\mathrm{O}\left(r^{t+1} / n^{t-1}\right)\right.$. The method by which we prove Theorem 3 yields in principle a complete asymptotic expansion of $J_{n}^{(t)}$ in descending powers of $n$, although the later terms are quite complicated. In the final section of our paper, we present a generalization of the first equality in Theorem 1.

\section{Discussion of Theorem 2}

We shall not give a proof of this theorem, since many are available. Indeed, using the Principle of Inclusion-Exclusion, the left side can be interpreted as the number of ways to color properly the complete graph $K_{n}$ with $X$ colors, which agrees with the right side. More generally, we may replace the graph $K_{n}$ on the left with an arbitrary graph $G$, and then on the right we replace the displayed polynomial with the chromatic polynomial of $G$. A good reference for this is [2]. 
Since the coefficients of $X(X-1) \cdots(X-n+1)$ are the (signed) Stirling numbers of the first kind, $s(n, k)$, Theorem 2 is equivalent to:

$$
\sum_{\substack{S \subseteq E_{n} \\ c\left(G_{S}\right)=k}}(-1)^{|S|}=s(n, k)
$$

In this form the theorem states that among graphs of $n$ vertices and $k$ connected components, the excess of the number with an even number of edges over those with an odd number of edges is the signed Stirling number of the first kind $s(n, k)$. The case $k=1$ of this interesting interpretation appeared as a Monthly Problem a few years ago, and in the solution the generalization to larger $k$ was noted, [3].

We close this section with a useful inclusion/exclusion enumeration formula based on Theorem 2.

Corollary. Let $X$ be a set of combinatorial objects which may have properties corresponding to the pairs $E_{n}, n \geq 1$. Suppose that for $S \subseteq E_{n}$, the number of objects which have at least all the properties of $S$ depends only on c $\left(G_{S}\right)$, the number of connected components of the graph $G_{S}$ determined by the pairs $S$. If this number is $f\left(\mathrm{c}\left(G_{S}\right)\right)$, then,

$$
\#\{x \in X: x \text { has no property }\}=\sum_{k=1}^{n} s(n, k) f(k) \text {. }
$$

\section{An Application}

We shall now use the above inclusion/exclusion formula to give another proof of the beautiful formula found by Boris Pittel [5]. The formula is

$$
M_{n}^{(t)}=e^{-t} \sum_{i_{1}=1}^{\infty} \cdots \sum_{i_{t}=1}^{\infty} \frac{\left(i_{1} \cdots i_{t}\right)_{n}}{i_{1} ! \cdots i_{t} !}
$$

where, again, $M_{n}^{(t)}$ is the number of $t$-tuples of partitions satisfying

$$
\pi_{1} \wedge \pi_{2} \wedge \cdots \wedge \pi_{t}=\{\{1\},\{2\}, \ldots,\{n\}\} .
$$

A striking feature of Pittel's formula is its resemblance to Dobinski's formula (see [6])

$$
B_{n}=e^{-1} \sum_{i=1}^{\infty} \frac{i^{n}}{i !}
$$

or its $t$-th power:

$$
\left(B_{n}\right)^{t}=e^{-t} \sum_{i_{1}=1}^{\infty} \cdots \sum_{i_{t}=1}^{\infty} \frac{\left(i_{1} \cdots i_{t}\right)^{n}}{i_{1} ! \cdots i_{t} !} .
$$

A collection of $t$ partitions will have nontrivial meet precisely when there is at least one pair of integers $i$ and $j$ which belong to the same block in all $t$ of the partitions. Let $X$ be the set of all $t$-tuples of partitions, and let $(i, j)$ be the property that when the meet 
of a $t$-tuple is formed, elements $i$ and $j$ are still in the same block. Then, by the Corollary of the previous section,

$$
M_{n}^{(t)}=\sum_{k=1}^{n} s(n, k)\left(B_{k}\right)^{t}
$$

Herb Wilf pointed out that the previous identity is equivalent to, (1). Indeed,

$$
\begin{aligned}
e^{-t} \sum_{i_{1}=1}^{\infty} \cdots \sum_{i_{t}=1}^{\infty} \frac{\left(i_{1} \cdots i_{t}\right)_{n}}{i_{1} ! \cdots i_{t} !} & =e^{-t} \sum_{i_{1}=1}^{\infty} \cdots \sum_{i_{t}=1}^{\infty} \frac{\sum_{k=1}^{n} s(n, k)\left(i_{1} \cdots i_{t}\right)^{k}}{i_{1} ! \cdots i_{t} !} \\
& =\sum_{k=1}^{n} s(n, k)\left(e^{-1} \sum_{i=1}^{\infty} \frac{i^{k}}{i !}\right)^{t} \\
& =\sum_{k=1}^{n} s(n, k)\left(B_{k}\right)^{t},
\end{aligned}
$$

proving the theorem.

\section{Proof of the First Equality in Theorem 1}

Since (see for example [1])

$$
\sum_{n \geq 0} s(n, k) \frac{x^{n}}{n !}=\frac{(\log (1+x))^{k}}{k !},
$$

equation (2) is equivalent to

$$
\left[\frac{x^{n}}{n !}\right] M_{t}(x)=\sum_{k \geq 0}\left(B_{k}\right)^{t}\left[\frac{x^{n}}{n !}\right] \frac{(\log (1+x))^{k}}{k !} .
$$

The linear operator $\left[\frac{x^{n}}{n !}\right]$, "take the coefficient of $\frac{x^{n}}{n !}$," can be moved outside the summation on the right. Then, we may drop the $\left[\frac{x^{n}}{n !}\right]$ from both sides, leaving an identity. The identity is exactly the first equality in Theorem 1, after substituting $e^{x}-1$ for $x$.

\section{$5 \quad$ Proof of the Second Equality in Theorem 1}

There is a Basic Principle of Exponential Generating Functions which says that if $J(x)$ is the egf of certain labeled combinatorial objects, then $\exp \{J(x)-1\}$ is the egf for partitions of $n$ with a $J$-object built on each block. A very good account of this exponential formula is given in [7], Chapter 3. It suffices, therefore, to establish a bijection

$$
\underbrace{\Pi_{n} \times \Pi_{n} \times \cdots \times \Pi_{n}}_{t \text { factors }} \longleftrightarrow Q_{n t}
$$

where $Q_{n t}$ consists of all sets of $t$-tuples of partitions

$$
\left\{\left(x_{11}, x_{12}, \ldots x_{1 t}\right), \ldots,\left(x_{\ell 1}, x_{\ell 2}, \ldots x_{\ell t}\right)\right\}
$$


with the property that each join

$$
x_{i 1} \vee x_{i 2} \vee \cdots \vee x_{i t}
$$

is a one-block partition $\left\{S_{i}\right\}$, where $S_{i} \subseteq[n]$ and $\left\{S_{i}: 1 \leq i \leq \ell\right\}$ is a partition of $[n]$. To repeat for clarity, each member of $Q_{n t}$ is a nonempty set (whose size is denoted here $\ell \geq 1$ ), each element of which is a $t$-tuple $\left(x_{i 1}, \ldots, x_{i t}\right)$. The various $x_{i j}$ are themselves partitions of a set $S_{i} \subseteq[n]$; the join (over $j$ ) of the $x_{i j}$ equals $\left\{S_{i}\right\}$; and $\pi=\left\{S_{i}: 1 \leq i \leq \ell\right\}$ is a partition of $[n]$.

Once the definition of the set $Q_{n t}$ has been comprehended, the bijection (3) with the Cartesian product $\left(\Pi_{n}\right)^{t}$ is fairly natural. In the direction $\longrightarrow$, let a $t$-tuple of partitions $\left(\pi_{1}, \ldots, \pi_{t}\right)$, be given. Let $\pi=\left\{S_{i}: 1 \leq i \leq \ell\right\}$ be their join. The partitions $x_{i j}$, $(1 \leq i \leq \ell, 1 \leq j \leq t)$, are the nonempty intersections of the blocks of $\pi_{j}$ with the set $S_{i}$.

In the other direction $\longleftarrow$, let $T$ be a set of the form (4), consisting of $t$-tuples of partitions $x_{i j}$. We know that each join $\vee_{j=1}^{t} x_{i j}$ is a one-block partition $\left\{S_{i}\right\}$. Since $x_{i j}$ is a partition of $S_{i}$, and $\left\{S_{i}: 1 \leq i \leq \ell\right\}$ is itself a partition of $[n]$, it follows that

$$
\pi_{j}=x_{1 j} \cup x_{2 j} \cup \cdots \cup x_{\ell j}
$$

is a partition of $[n]$. The $t$-tuple $\left(\pi_{1}, \pi_{2}, \ldots \pi_{t}\right)$ so formed is the one to be associated by the bijection with the initially given set $T$.

\section{Calculations}

The equation (2) yields efficient calculation of $M_{n}^{(2)}$. By differentiating the second equality of Theorem 1, we obtain, by a familiar technique, the recursion

$$
J_{n+1}^{(t)}=\left(B_{n+1}\right)^{t}-\sum_{j=1}^{n}\left(\begin{array}{l}
n \\
j
\end{array}\right)\left(B_{j}\right)^{t} J_{n-j+1}^{(t)}, \quad n \geq 0,
$$

and this permits efficient calculation of $J_{n}^{(t)}$. By these means we determine the following table for $t=2$.

$\begin{array}{crr}n & M_{n}^{(2)} & J_{n}^{(2)} \\ 0 & 1 & 1 \\ 1 & 1 & 1 \\ 2 & 3 & 3 \\ 3 & 15 & 15 \\ 4 & 113 & 119 \\ 5 & 1153 & 1343 \\ 6 & 15125 & 19905 \\ 7 & 245829 & 369113 \\ 8 & 4815403 & 8285261 \\ 9 & 111308699 & 219627683 \\ 10 & 2985997351 & 6746244739\end{array}$




\section{$7 \quad$ Proof of Theorem 3}

To simplify and avoid proliferation of cases, we take $t=2$ and accuracy $n^{-2}$; the method can be adapted for any fixed $t \geq 2$, and any desired accuracy. It is an iterative method, and we need an initial estimate. From [5] we know

$$
J_{n}^{(2)}=\left(B_{n}\right)^{2}\left(1+\mathrm{O}\left(r^{3} / n\right)\right),
$$

where $r$ is the positive real solution of $r e^{r}=n$. By the Moser-Wyman method [4] we have

$$
\frac{B_{n+1}}{B_{n}}=\frac{n+1}{r}\left(1+\mathrm{O}\left(n^{-1}\right)\right)
$$

and from the recursion (5),

$$
\frac{J_{n+1}^{(2)}}{\left(B_{n+1}\right)^{2}}=1-n \frac{J_{n}^{(2)}}{\left(B_{n+1}\right)^{2}}-\frac{1}{\left(B_{n+1}\right)^{2}} \sum_{j=2}^{n}\left(\begin{array}{c}
n \\
j
\end{array}\right)\left(B_{j}\right)^{2} J_{n+1-j}^{(2)} .
$$

We bound the summation above by replacing $J_{n+1-j}^{(2)}$ with $\left(B_{n+1-j}\right)^{2}$. The resulting convolution can be further bounded as in the proof of Theorem 5 in [5]; namely, it is the terms at the extreme ends of the sum which dominate:

$$
\frac{1}{\left(B_{n+1}\right)^{2}} \sum_{j=2}^{n}\left(\begin{array}{l}
n \\
j
\end{array}\right)\left(B_{j} B_{n+1-j}\right)^{2}=\mathrm{O}\left(r^{4} / n^{2}\right) \text {. }
$$

With

$$
\frac{J_{n}^{(2)}}{\left(B_{n+1}\right)^{2}}=\frac{J_{n}^{(2)}}{\left(B_{n}\right)^{2}}\left(\frac{B_{n}}{B_{n+1}}\right)^{2}
$$

the bound for the summation, (6), and (7) we have

$$
\frac{J_{n}}{\left(B_{n}\right)^{2}}=1-\frac{r^{2}}{n}+\mathrm{O}\left(r^{5} / n^{2}\right)
$$

(When we replace $n$ by $n-1$, we must replace $r$ by $r+\mathrm{O}\left(n^{-1}\right)$.) We now repeat the process. This time we substitute into

$$
\begin{aligned}
\frac{J_{n+1}}{\left(B_{n+1}\right)^{2}} & =1-n \frac{J_{n}^{(2)}}{\left(B_{n+1}\right)^{2}}-4\left(\begin{array}{c}
n \\
2
\end{array}\right) \frac{J_{n-1}^{(2)}}{\left(B_{n+1}\right)^{2}}-J_{1}^{(2)}\left(\frac{B_{n}}{B_{n+1}}\right)^{2} \\
& -\frac{1}{\left(B_{n+1}\right)^{2}} \sum_{j=3}^{n-1}\left(\begin{array}{l}
n \\
j
\end{array}\right)\left(B_{j}\right)^{2} J_{n+1-j}^{(2)},
\end{aligned}
$$

using in place of $(7)$ the more accurate

$$
\frac{B_{n+1}}{B_{n}}=\frac{n+1}{r}\left(1-\frac{2+4 r+r^{2}}{2(r+1)^{2} n}+\mathrm{O}\left(r^{2} / n^{2}\right)\right),
$$

and

$$
\frac{1}{\left(B_{n+1}\right)^{2}} \sum_{j=3}^{n-1}\left(\begin{array}{l}
n \\
j
\end{array}\right)\left(B_{j} B_{n+1-j}\right)^{2}=\mathrm{O}\left(r^{6} / n^{3}\right)
$$

The result, after some algebra, including this time a replacement of $n$ by $n-1$ and of $r$ by $r-r /(1+r) n+\mathrm{O}\left(n^{-2}\right)$, is the formula stated as Theorem 3 . 


\section{A Generalization in Terms of Whitney Numbers}

In the lattice $\Pi_{n}, 0$ is the finest partition $\{\{1\}, \ldots,\{n\}\}$, and 1 is the coarsest $\{\{1, \ldots, n\}\}$. The intervals of $\Pi_{n}$ have an interesting recursive structure. Consider first an interval of the form $[\pi, 1]$ Observe that the latter interval is isomorphic to $\Pi_{k}$, where $\pi$ has $k$ blocks. Now, if we take any $t$-tuple of partitions, and form their meet, we obtain some partition $\pi$. Thus, we can count all $t$-tuples according to their meet, as follows:

$$
\left(B_{n}\right)^{t}=\sum_{k} S(n, k) M_{k}^{(t)}
$$

This provides, by inversion, another proof and further understanding of equation 2 . We can formalize this as follows.

Theorem 4 Let $L_{n}$ be a sequence of lattices with rank $(1)=n$. Assume that each interval $[x, 1] \subseteq L_{n}$ is isomorphic to $L_{k}$ if $x \in L_{n}$ and $\operatorname{rank}(x)=n-k$. If $M_{L}^{(t)}{ }_{n}$ equals the number of t-tuples of points in $L_{n}$ whose meet is 0 , then

$$
\left|L_{n}\right|^{t}=\sum_{k} W_{n-k} M_{L k}^{(t)}
$$

where $W_{k}$ are the Whitney numbers of the second kind, the number of elements of rank $k$.

As an example, consider the lattice $B_{n}$ of subsets of $[n]$. Theorem 4 tells us

$$
2^{n t}=\sum_{k}\left(\begin{array}{l}
n \\
k
\end{array}\right) M_{B k}^{(t)} .
$$

By inversion, we conclude there are

$$
\sum_{k}(-1)^{k}\left(\begin{array}{l}
n \\
k
\end{array}\right) 2^{t k}=\left(2^{t}-1\right)^{n}
$$

$t$-tuples of subsets of $[n]$ whose intersection is empty.

A similar remark can be made for the join operation in $\Pi_{n}$. Namely, the interval $[0, \pi]$ is isomorphic to a Cartesian product of $\lambda_{1}$ copies $\Pi_{1}$ with $\lambda_{2}$ copies $\Pi_{2}$, etc., where the shape of partition $\pi$ is $1^{\lambda_{1}}, \ldots n^{\lambda_{n}}$. Hence,

$$
\left(B_{n}\right)^{t}=\sum_{\lambda \vdash n} \frac{n !}{\prod_{i}(i !)^{\lambda_{i}} \lambda_{i} !} \prod_{i}\left(J_{i}^{(t)}\right)^{\lambda_{i}}
$$

In this equation, the fraction on the right is the well known [1] formula for the number of partitions of shape $\lambda$. This identity is equivalent to the second equality of Theorem 1 . We will not formulate a generalization, since no examples other than $\Pi_{n}$ come to mind!

Acknowledgment. I would like to thank Konrad Engel, Ira Gessel, and David Jackson for helpful comments on earlier versions of the paper. 


\section{References}

[1] Louis P. Comtet, Advanced Combinatorics, D. Reidel, Dordrecht, Holland, 1974.

[2] László Lovász, Combinatorial Problems and Exercises, Problem 9.37, p.63, Elsevier North-Holland, NY, (1979).

[3] Paresh J. Malde, Allen J. Schwenk, Stephen C. Locke, Herbert S. Wilf, Bruce E. Sagan, Richard Holzsager, The difference between graphs of even and odd size, Problem Solution, American Mathematical Monthly 101 (1994) 686-687.

[4] L. Moser and M. Wyman, An asymptotic formula for the Bell numbers, Trans. Royal Soc. Canada III 49 (1955) 49-54.

[5] Boris Pittel, Where the typical set partitions meet and join, Electronic Journal of Combinatorics 7 (2000), R5, 15 pages.

[6] Gian-Carlo Rota, The number of partitions, American Math. Monthly 71 (1964), 498-504.

[7] Herbert S. Wilf, generatingfunctionology, second edition, Academic Press, San Diego, (1994). 